\title{
Pediatric palliative care and surgery
}

\author{
Katherine C. Ott ${ }^{1}$, Teresa M. Vente ${ }^{2}$, Timothy B. Lautz ${ }^{1}$, Elisha D. Waldman ${ }^{2}$ \\ ${ }^{1}$ Division of Pediatric Surgery, Ann and Robert H. Lurie Children's Hospital, Chicago, IL, USA; ${ }^{2}$ Division of Palliative Care, Ann and Robert H. \\ Lurie Children's Hospital, Chicago, IL, USA \\ Contributions: (I) Conception and design: All authors; (II) Administrative support: All authors; (III) Provision of study materials or patients: All \\ authors; (IV) Collection and assembly of data: KC Ott, TM Vente; (V) Data analysis and interpretation: All authors; (VI) Manuscript writing: All \\ authors; (VII) Final approval of manuscript: All authors. \\ Correspondence to: Katherine C. Ott. 225 E. Chicago Ave, Chicago, IL 60611, USA. Email: kott@luriechildrens.org.
}

\begin{abstract}
Care for pediatric patients with serious or potentially life-limiting illness involves the interplay of multiple medical and surgical teams within the hospital. Pediatric surgeons are capable of performing procedures that can improve the quality of life for children facing serious illness, but which also carry the potential for significant risk and burden. Patients and families are often faced with decisions about invasive surgical procedures and interventions, stressing the need for seamless collaboration between palliative care and surgical providers. Equally important is the need for clear and open-ended communication with patients and families by all medical teams to determine if potential surgical procedures and interventions align with their goals and to ensure that the perceived benefits of interventions outweigh any risks. Over the last two decades, pediatric palliative care has grown into a thriving medical subspecialty Despite the importance of collaborative care, there is lack of literature on the interaction of pediatric surgery and palliative care and the role of pediatric surgeons in providing primary palliative care. This review defines surgical pediatric palliative care, and provides an in-depth discussion of the unique complexities involved in caring for children with serious and potentially life-limiting illness, while highlighting specific challenges through detailed case presentations.
\end{abstract}

Keywords: Chronic illness; pediatric palliative care; pediatric surgery; surgical palliative care

Submitted Dec 01, 2020. Accepted for publication May 26, 2021.

doi: 10.21037/apm-20-2370

View this article at: http://dx.doi.org/10.21037/apm-20-2370

\section{Introduction}

Pediatric surgeons are often involved in the ongoing treatment of patients with life-limiting conditions. Decisions concerning when or even if a patient should undergo surgery are extremely difficult for patients and their families to navigate. In 2000, the American Academy of Pediatrics (AAP) provided recommendations regarding pediatric palliative care, however the role of surgery in this context was not defined (1). There is very limited literature that specifically addresses the role of the pediatric surgeon in the palliative care team despite the fact that pediatric surgeons are often called on to evaluate and perform interventions in the context of palliative care. This includes performing procedures that could improve the quality of life for children facing serious illness, but which also may carry the potential for significant risk and burden. Evaluating to what extent procedures align with child and family goals is therefore critical.

As hospital systems, technologies, interventions, and the needs of patients become more complex, excellent communication and care coordination between specialized teams is more important than ever. Communication can present challenges and pitfalls, but also opportunities for better collaboration, in particular between surgeons and palliative care providers. Communication and care coordination are pivotal cornerstones of the specialized expertise that falls within the scope of pediatric palliative care providers. Quality palliative care is critically important to the patient and surgeon relationship and must be taken 
into account in decision making (2).

\section{Existing data on pediatric surgical palliative care}

Almost 15 million (or $19.8 \%$ ) of all children in the US have special healthcare needs, with a smaller but significant number having complex chronic illness (3). At least 55,000 children die each year, with half dying of chronic disorders (4). Medical and technological advances have allowed children with chronic conditions to live longer than ever before. This includes advances in diagnostics, treatments, and supportive care, and applies across a wide range of childhood conditions including cancer, cystic fibrosis, neurodegenerative conditions, and a host of complex chronic conditions. In a study from 2008 of six PPC teams in North America, 515 patients who received services were followed up for a year with an overall survival rate of $69.7 \%$ (5). In line with this, palliative care services for pediatric patients have been steadily increasing in number in the past two decades. In this setting, surgery has a supportive actor role integrated with other aspects of the palliative care team.

Recent publications have highlighted the magnitude of healthcare utilization and high-intensity end-of-life care for children with cancer $(6,7)$. The majority of these patients receive palliative treatment during the last months of life, including different and/or multiple surgical procedures. Of children who die in the hospital with cancer, $35 \%$ will receive anesthesia for a surgical or medical procedure $(7,8)$. A majority of these procedures involve intravenous access, feeding access, or tracheostomy placement for mechanical ventilation. Despite advances in pediatric palliative care, parents report that they do not feel that their children's symptoms are being controlled well at the end of life (9-13). One study highlighted the magnitude and often short time from invasive procedures to the time of death experienced by some children (14).

It can be challenging for physicians and families to come to terms with a serious or potentially life-limiting diagnosis in a child; however, once this occurs, elements of palliative care are more likely to be initiated and have been shown to reduce the number of intensive end-of-life measures $(15,16)$. Physicians and nurses were surveyed about barriers to advanced palliative care discussions in pediatrics and showed that $71 \%$ felt that these discussions happened too late (17). In fact, $92 \%$ believed these discussions should be initiated at the time of diagnosis or during a time of stability but that $60 \%$ of the time, discussions were only initiated when death was near. Intensive medical care in the last weeks of a life has been shown to cause significant stress, increased rates of depression, and negatively impact the quality of life of family members of the patient (15). In addition, these procedures can keep a child in the hospital despite recognizing the child's home as a preferred place of death for many patients and their families $(6,18,19)$. Clinicians often avoid discussions of palliative care because they do not know how to initiate the discussion and don't want families to lose hope (17). It is imperative that surgeons are trained in initiating these discussions where appropriate and have an open line of communication with the palliative care team in order to determine what is right for each patient and their family.

\section{General concepts in pediatric palliative care}

Over the last two decades, pediatric palliative care has grown into a thriving medical subspecialty (20). Each year sees the introduction of new ACGME-accredited fellowship programs, completion of which is now a requirement for taking the Hospice and Palliative Medicine board exam for professional certification. Pediatric palliative care practice changes have been influenced by policy statements published by national medical associations to emphasize a commitment to palliative care principles for pediatric patients and provide guidelines for care $(1,21,22)$.

The underlying philosophy of palliative care for all patients, irrespective of age, is largely the same: to promote an interdisciplinary approach to support for patients and families facing serious, potentially life-limiting illness regardless of stage or prognosis. This often includes recommendations around symptom management, but, more crucially, involves ongoing exploration of hopes, fears, and values in order to better understand a patient's and family's goals of care in order to make sure that treatment options offered align with those goals.

Though those principles are common to adult and pediatric palliative care, it is important to distinguish the features and challenges of palliative care unique to pediatric patients. Epidemiology of pediatric illness differs from that of adults with majority of childhood deaths occurring from congenital anomalies, cardiac defects and malignant neoplasms (23). While many tend to think about pediatric palliative care in the context of these pediatric oncologic and progressive diseases, in reality a majority of children in the U.S. referred to pediatric palliative care services 
are children with complex chronic illness, often genetic, metabolic, or neurologic disorders with uncertain disease trajectories. Many of these children with life-limiting conditions are living longer lives than they may have in the past due to better supportive care and new interventions. As a result, guidelines for pediatric providers recommend early involvement of palliative care with referral at the time of diagnosis of serious illness, so that palliative care can be delivered as part of a concurrent package along with diseasedirected therapies (24). The plan of care for children with potentially life-limiting illness can occur along a spectrum that includes prevention, treatment, curative measures or life-prolonging interventions in combination with palliative care $(21,25)$. Because of this, involvement with palliative care services may change over time as goals of care evolve.

In children with complex and serious illness, multiple subspecialty providers are likely to be involved. This creates a particular risk for miscommunication and fragmented care, as well as for prolonged hospital stays, greater use of urgent care services, and greater morbidity. For optimal multimodal care, subspecialists must have a collaborative approach with an emphasis on excellent, centralized communication $(21,24)$. Subspecialists may offer a range of medical therapies, surgical interventions, medical technologies or rehabilitation measures with aims ranging from cure to comfort. Every diagnostic or treatment intervention offered by the medical team should be considered in the context of patient and family values, and realistic expectations of outcomes of treatment should be discussed $(1,2)$. With the aim of improving quality of life of, the level of function and disability, pain and suffering, or other possible burdens must be considered for each intervention and treatment offered (26).

Paramount to palliative care is the relief of suffering. The expression of physical symptoms, as well as emotional and spiritual distress changes and evolves over the course of childhood development. The World Health Organization as well as national medical associations recognize the importance of an interdisciplinary approach to palliative care to address multiple domains of distress including physical, emotional, social and spiritual (27). Within pediatric palliative care, interdisciplinary providers must be able to account for differences in distress across childhood but also within the context of a family unit and provide support for families during pediatric illness and after a child's death.

One of the most fundamental tenets of pediatric palliative care is the recognition that there are developmental differences across a child's life span that impact many features of care including diagnosis and prognosis, communication and decision making, treatments and therapies (21). Methods of communication by the medical team must be tailored to a child's level of development (25). Children will vary in their ability to participate in healthcare decisions based on age and health literacy (28-30). To the extent that children can participate in medical decision making, children should be included in discussions regarding treatments and interventions.

The basic concepts of palliative care are essential not only for pediatric palliative care specialists but also for generalists, interventionalists, subspecialists and surgeons. Early integration of palliative care concepts helps to ensure that palliative care is integrated and maintained as part of the continuum of care for children with serious and lifethreatening illness (24).

\section{Defining pediatric surgical palliative care}

Surgical palliation involves undergoing a procedure or intervention which involves a certain amount of morbidity and risk in order to alleviate specific symptoms. Palliative surgery can be ethically prohibited, ethically mandatory, or ethically allowable, depending on the goals of the patient and their family (31). The primary objectives of surgical palliation include relief of symptoms, prolonging life through the restoration of organ function and/or improvement in quality of life. It is important to note that the quality of life for the patient must be defined by the patient and family and not by the medical or surgical team. The palliative care team can help the family define their goals in regards to quality of life. The surgical team can work together with both the palliative care team and the family to weigh the risks against these goals. Often the focus is on decreased symptom burden, maintaining level of function or ongoing ability to participate in activities that bring joy and fulfillment. These benefits should be balanced against overall disease burden, immunodeficiency, the ability to heal, infection and bleeding risks, patient performance status, duration of hospitalization, surgical morbidity and mortality, anticipated survival, and need for additional palliative measures. If a procedure provides no benefit to the patient, there is no ethical obligation to offer it to a family. Each procedure no matter how small carries with it certain risks. In the event that a procedure provides no benefit to the patient and only potential risks, the surgeon is not only not obligated to offer the procedure 
but also not justified in doing so. A comprehensive palliative care plan is developed through multidisciplinary discussion of the patient's condition and of the natural history of the disease (the latter of which, as we have noted, can be especially challenging in many complex chronic illnesses of childhood).

In all of this process, the pediatric surgeon is not merely a technician. It is the surgeon's responsibility as well as that of the rest of the care team to ensure as best as possible that the patient and family have a clear comprehension of the options and possible outcomes. Surgeons may become involved with the care of seriously ill children in several ways, which may impact the extent to which the surgeon is able to build a trusting relationship. In some cases, the surgeon may have a well-established relationship with the patient after having provided care earlier in the course of disease. In these situations, they may be well positioned to help with the ongoing exploration of goals, especially if an underlying disease is progressing in a way that might impact those goals. The surgeon may have an important role in helping a family weigh the risks and benefits of additional surgical interventions; certainly, the surgical team often understands the technical risks and potential benefits of surgical interventions better than other medical teams might, and are therefore critical to evaluating whether those interventions align with family goals. Special consideration might be given to whether a procedure is reversible and what effect this may have on weighing risks and benefits. While many procedures technically can be reversed the likelihood of this actually occurring varies considerably between patients depending on the disease state, indication and prognosis which must be discussed with the patient and their family.

Of course, surgeons may also be consulted for a procedure in a situation where they may not have an established relationship with a patient or family. In these cases, exploration of goals of care and evaluation of a family's understanding of the treatment options might be more challenging than when there is a preexisting relationship. Under these circumstances, surgeons need to rely on a combination of their own primary palliative care skills to communicate with the family, as well as on excellent collaborative relationships with other teams that might already know the family, such as the primary team or the palliative care team. Correctly or not, the surgeon may be viewed by a family as the last hope to regain some sense of normalcy, if not to actually effect a cure. The burden then falls primarily (but not solely) on the surgeon to explore hopes and expectations, as well as to communicate possible risks and benefits in the context of those hopes. It is important to remember, of course, that a procedure may itself result in increased suffering or burden on a patient and family. Frequently, one hears from a family phrases like "we want everything done". But that idea of "everything" is deceptively complex, and should never be taken at face value; rather, the idea of "everything" must be explored in the context of a family's goals, so that surgeons, along with other clinicians, can best evaluate when an intervention might not align with those goals. At times, the service rendered may well be to determine that a given intervention is best not pursued, or even that it makes the most sense to place limits on interventions or postoperative treatments.

\section{Unique aspects of pediatric surgical palliative care compared to adults}

There are some unique challenges around palliative surgery in the pediatric population compared to adults. First and foremost, the prognosis and expected course of illness is frequently more difficult to determine in pediatric complex chronic illnesses than it is in diseases more commonly seen in adults (2). Pediatric surgeons are uniquely rewarded by the patient's potential of a full and productive life, which may not be the immediate goal in palliative surgery. When a child presents with a request for palliative treatment, the goals of the surgeon must adjust to meet the situation at hand. One important consideration is when and how to apply the label of "palliative" to a procedure. Is that defined by the patient's prognosis? Is it defined by the clinicians? By the family? Labelling a procedure as "palliative" may be less important than just the process of making sure that the risks and likely outcomes of a procedure align with a patient and family's goals. The difficulty in ascertaining expected timelines can add significantly to the stress of decision making. One recent study showed that a large number of children undergo surgery during their terminal hospital admission and that $20 \%$ of those die within 24 to 48 hours of their operation, which is significantly higher than for adults (14). It is important to understand to what extent those families had prior exposure to goals of care discussions and possibly even palliative care services, though the current literature is lacking.

Given the difficulty in ascertaining prognosis for so many children with complex chronic illness, even the very definition of when a surgical intervention is considered "palliative" may be hard to pin down. A range of procedures 
could be considered palliative in a given patient. These include interventions which may be intended to control symptoms such as pain, bleeding, shortness of breath, or bowel obstruction. Additional non-curative procedures such as vascular access or gastrotomy may provide for the administration of treatment and/or artificial nutrition and hydration. Each of these procedures brings with it a specific set of risks that must be discussed with the patient and family. For example, children need to undergo anesthesia more often than adults even for small procedures. This is not without its own risks. Surgeons may need to consider other additional pain control methods postoperatively. Parents may want to avoid any possibility of additional pain or suffering to their child and decline procedures despite the chance of some degree of benefit.

Conversely, parents may demand that procedures be performed that carry significant risk in order to obtain a relatively small benefit. Futility, however, may be a somewhat subjective idea. There may be psychological or spiritual benefits that the surgeon is not considering. When it feels like there might be a disconnect between the surgeon and the family or the patient, the palliative care team may help to delineate family goals and communicate with surgeons to determine if those goals reasonably align with the procedure. Additionally, the issue of resuscitation status (i.e., DNR orders) must be discussed with the entire team including the surgeon and anesthesiologist providing care during the procedure. If the surgeons do not already have a preexisting relationship with the family, working closely with those medical team members who do (be it the primary team or palliative care team) is essential in order to best frame the discussion. Those teams may also provide valuable guidance to the surgeon on the specific goals for the requested procedure. When expectations are not met or goals are not clearly defined, conflicts can result. Surgical palliation should be discussed within a comprehensive multidisciplinary palliative care plan to achieve the best outcomes and maximize patient satisfaction. In some instances, ethics consultations may also provide some clarity, in particular where there seems to be disagreement between teams and family or among family members (1).

The decision to potentially withhold or withdraw life prolonging therapies is, needless to say, excruciating for families. The surgeon, primary care team, and palliative care team can help the patient and their family through a model of shared decision-making, which allows for family and patient autonomy while not placing responsibility squarely on their shoulders alone (32). The recommendations should focus on family and patient values, hopes, and fears. Discussions should be centered around what is being done to make the patient more comfortable rather than what is not being done. When possible, the child should be included in these discussions in an age-appropriate manner; child life and similar services may prove very helpful allies in these efforts. Some parents may wish to not explain the prognosis to the child for fear that this may cause them to lose hope. Children often want to know more and be a part of their care, however, and may feel even more alone if they do not have someone to talk to about it $(21,30)$. There may well be parents who prefer to not discuss death with their children, and for whom that is the right choice, but there are others who may end up regretting that choice. Primary palliative care skills, whether utilized by the surgical team, the primary team, or the palliative care team, are essential in helping determine which family is which and in meeting the child's needs regardless. The surgeon may serve an additional, important role in this process by discussing how procedures may provide comfort to the child or how they may be harmful.

\section{Case examples}

\section{Time-limited trial}

A 10-year-old male with recurrent ALL is enrolled in a clinical trial for cart-T cell therapy. He develops severe cytokine release syndrome (CRS), progressing to high fever, vasopressor-requiring circulatory shock, pulmonary edema and respiratory failure. Despite supportive care measures, including mechanical ventilation, as well as systemic therapy with tocilizumab and corticosteroids, he progresses to refractory hypoxemia. The pediatric surgery team is consulted to determine eligibility for Extracorporeal Membrane Oxygenation (ECMO).

In discussion with the primary oncology team, it is indicated that early results with car-T therapy support a high rate of achieving complete remission, but that the long-term survival of patients with this condition remains unknown. The oncology, surgery, palliative care, and PICU teams arrange for a family meeting with the patient's parents. The care team explains the critical nature of the patient's condition, and discusses the risk of major complications with ECMO, including bleeding and stroke. The team cites data from the ELSO registry indicating a $40-60 \%$ change of survival to discharge for a patient undergoing ECMO for shock and ARDS. The oncology 
team again stresses the promising nature of car- $\mathrm{T}$ cell therapy but uncertain long-term prognosis.

The parents indicate a clear understanding of the critical nature of his current condition, realistic impression of the risks and benefits of ECMO and appreciation for the long-term uncertainty with his oncologic condition. They continue to express hope for their son's recovery and a desire to pursue all medical interventions so long as there is a realistic hope of long-term survival with meaningful recovery. They request that the patient be placed on ECMO, and that another care conference be initiated if/ when there is any change in his condition which might alter his realistic likelihood of meaningful recovery. This strategy provides the patient and family with a time-limited trial which can help them make future decisions $(33,34)$.

\section{Palliative care and congenital cardiac surgery}

A baby is born to a 38-year-old G1P1 mother. Prenatal imaging was consistent with congenital heart disease and maternal cell-free DNA was consistent with trisomy 13. Postnatal evaluation confirms the diagnosis of trisomy 13 and tetralogy of Fallot (TOF). The baby does well in the neonatal period and the family comes to meet with the cardiology and cardiac surgery teams at 3 months of age to discuss potential repair.

Prior to meeting with the parents, the cardiology and $\mathrm{CV}$ surgery teams meet and discuss reluctance to perform complex cardiac surgery with a real risk of serious surgical morbidity and mortality in a patient with limited life expectancy due to the underlying trisomy 13 . The $\mathrm{CV}$ surgery APN suggests involving the palliative care team, who had previously met the family in the NICU.

The CV surgery team explains the anatomy of the baby's cardiac defect, the complexity of the surgical repair, as well as the short- and long-term morbidity and mortality of surgery. They indicate that many families of children with life-limiting genetic defects opt to forgo putting their child through such a procedure.

The parents indicate an understanding of the severity of their daughter's congenital anomalies and an acceptance that her trisomy is life-limiting. However, they express hope about recent improvements in the life expectancy of children with this condition and a desire to spend as much quality time with their child as possible. They explain that they were through several attempts at IVF in order to conceive a child and a desire to maximize the amount of time they can spend at home with their daughter free of medical devices such as supplemental oxygen. After careful multidisciplinary discussion, the team arranges for repair of her TOF with ongoing palliative care follow-up to assist with symptom management in the postop period and the longer-term consequences of her underlying illness.

\section{When surgery provides no benefit}

A 17-year-old male presents with a second relapse of alveolar rhabdomyosarcoma. His initial tumor in the right thigh was treated with surgery and radiation. One month after completion of his initial therapy he developed pulmonary relapse, treated with additional chemotherapy and whole lung radiation. His disease remained initially stable, but after completion of chemotherapy for his relapsed disease, he developed back pain and underwent imaging including a whole-body PET-CT which demonstrated innumerable lung lesions and multiple sites of bony involvement.

His primary oncologist explains the imaging findings and conveys to the patient and family that while his disease can no longer be cured, there are options to potentially slow progression of his disease, as well as therapies to minimize pain and optimize his quality of life. He is starting on a phase I trial but requests to meet with surgery to discuss pulmonary metastasectomy.

The patient and surgeon have an established relationship from prior procedures (venous port placement and sentinel lymph node biopsy). The patient comes to see the surgeon with his parents and expresses an understanding of the terminal nature of his condition, but insists that "I just can't give up. I want to do absolutely everything possible to beat this cancer". Together they review the imaging, including the multiple, bilateral pulmonary nodules as well as the extra-pulmonary metastatic disease. The surgeon expresses empathy for the patient's current condition and understanding about his desire to do everything possible. However, she also explains that it is not possible to surgically remove all sites of metastatic disease and that there is no proven survival benefit from removal of some but not all sites of disease. The surgeon, oncology team, and palliative care team then meet with the family to collaboratively create a plan for ongoing, albeit limited, disease-directed interventions as well as plans for excellent symptom control. 


\section{Transition to end-of-life care}

An almost 1-year-old boy is being followed for progressive Tay-Sachs disease. He has been progressively losing motor skills, and is now having feeding difficulties, requiring NG supplementation for adequate nutrition and hydration. The surgeon has been consulted regarding the possibility of $g$-tube placement. His care team believes that a GT will provide durable feeding access and allow the family a safe and effective way to care for their child at home. His neurologist indicates that while it is rare for a child with his condition to live beyond 3-4 years of age, maintaining optimized nutrition can help maximize his survival duration.

When the family declines surgical intervention, his care team initially believes that there is a knowledge gap wherein the family is failing to fully understand the advantages of establishing durable feeding access. They arrange for a care conference with the family, including the neurologist, surgeon and palliative care team. During that discussion, the palliative care team guides the conversation towards establishing a better understanding of the parents hopes and desires for their child during his limited future months or years of life. The family indicates that the duration of his survival is not their primary concern. Rather, their aim is to keep him home, comfortable, well-loved and "nonmedicalized" for the extent of their time with him.

In conjunction with the primary team and palliative care, the surgeon explores what the family is hoping for, and guides a discussion of potential advantages of a g-tube versus potential surgical risks. After consideration of all factors the family decides not to risk g-tube placement, instead relying on the primary team and palliative care service, along with community-based hospice providers, to support their child for as long as possible without surgeries or invasive procedures.

\section{Conclusions}

With ongoing improvements in technology and interventions, the number of children living with complex chronic illness has increased greatly in recent years. However, along with increased lifespan and increased options for interventions has come increased complexity around decision-making. Now, more than ever, ongoing evaluation of patient and family hopes, values, and goals is central to providing the best care possible. Surgeons play a unique role in assessing family and child goals as they pertain to the risks and benefits of surgical interventions and thus should have the training and confidence in primary palliative care skills. Excellent communication and collaboration between primary medical teams, palliative care and surgical teams is paramount to providing comprehensive palliative care to pediatric patients and their families.

\section{Acknowledgments}

Funding: None.

\section{Footnote}

Provenance and Peer Review: This article was commissioned by the Guest Editors (Fabian M. Johnston and Ana Berlin) for the series "Palliative Care and Surgery" published in Annals of Palliative Medicine. The article has undergone external peer review.

Conflicts of Interest: All authors have completed the ICMJE uniform disclosure form (available at https://apm. amegroups.com/article/view/10.21037/apm-20-2370/coif). The series "Palliative Care and Surgery" was commissioned by the editorial office without any funding or sponsorship. The authors have no other conflicts of interest to declare.

Ethical Statement: The authors are accountable for all aspects of the work in ensuring that questions related to the accuracy or integrity of any part of the work are appropriately investigated and resolved.

Open Access Statement: This is an Open Access article distributed in accordance with the Creative Commons Attribution-NonCommercial-NoDerivs 4.0 International License (CC BY-NC-ND 4.0), which permits the noncommercial replication and distribution of the article with the strict proviso that no changes or edits are made and the original work is properly cited (including links to both the formal publication through the relevant DOI and the license). See: https://creativecommons.org/licenses/by-nc-nd/4.0/.

\section{References}

1. American Academy of Pediatrics. Committee on Bioethics and Committee on Hospital Care. Palliative care for children. Pediatrics 2000;106:351-7.

2. Shelton J, Jackson GP. Palliative care and pediatric surgery. Surg Clin North Am 2011;91:419-28, ix. 
3. Child and Adolescent Health Measurement Initiative. Who Are Children with Special Health Care Needs (CSHCN). Data Resource Center, supported by Cooperative Agreement 1-U59-MC06980-01 from the U.S. Department of Health and Human Services, Health Resources and Services Administration (HRSA), Maternal and Child Health Bureau (MCHB); Revised 4/2/12. 2012. Available online: www.childhealthdata.org

4. Calabrese CL. ACT--for pediatric palliative care. Pediatr Nurs 2007;33:532-4.

5. Feudtner C, Kang TI, Hexem KR, et al. Pediatric palliative care patients: a prospective multicenter cohort study. Pediatrics 2011;127:1094-101.

6. Johnston EE, Alvarez E, Saynina O, et al. Disparities in the Intensity of End-of-Life Care for Children With Cancer. Pediatrics 2017;140:e20170671.

7. Ananth P, Melvin P, Feudtner C, et al. Hospital use in the last year of life for children with life-threatening complex chronic conditions. Pediatrics 2015;136:938-46.

8. Zimmermann K, Cignacco E, Engberg S, et al. Patterns of paediatric end-of-life care: a chart review across different care settings in Switzerland. BMC Pediatr 2018;18:67.

9. Feudtner C. Collaborative communication in pediatric palliative care: a foundation for problemsolving and decision-making. Pediatr Clin North Am 2007;54:583-607.

10. Goldman A, Heller KS. Integrating palliative and curative approaches in the care of children with life-threatening illnesses. J Palliat Med 2000;3:353-9.

11. Kon AA, Ablin AR. It's not palliative care, it's palliative treatment. Lancet Oncol 2009;10:106-7.

12. Himelstein BP, Hilden JM, Boldt AM, et al. Pediatric palliative care. N Engl J Med 2004;350:1752-62.

13. Wolfe J, Grier HE, Klar N, et al. Symptoms and suffering at the end of life in children with cancer. N Engl J Med 2000;342:326-33.

14. Corkum KS, Lautz TB, Hebal FN, et al. Procedural burden experienced by children with cancer during their terminal hospital admission. J Pediatr Surg 2019;54:133-9.

15. Wright AA, Zhang B, Ray A, et al. Associations between end-of-life discussions, patient mental health, medical care near death, and caregiver bereavement adjustment. JAMA 2008;300:1665-73.

16. Keele L, Keenan HT, Sheetz J, et al. Differences in characteristics of dying children who receive and do not receive palliative care. Pediatrics 2013;132:72-8.

17. Durall A, Zurakowski D, Wolfe J. Barriers to conducting advance care discussions for children with life-threatening conditions. Pediatrics 2012;129:e975-82.

18. Mack JW, Chen K, Boscoe FP, et al. High intensity of end-of-life care among adolescent and young adult cancer patients in the New York State Medicaid Program. Med Care 2015;53:1018-26.

19. Gao W, Verne J, Peacock J, et al. Place of death in children and young people with cancer and implications for end of life care: a population-based study in England, 1993-2014. BMC Cancer 2016;16:727.

20. Sisk BA, Feudtner C, Bluebond-Langner M, et al. Response to Suffering of the Seriously Ill Child: A History of Palliative Care for Children. Pediatrics 2020;145:e20191741.

21. Behrman RE, Field MJ. When children die: Improving palliative and end-of-life care for children and their families. Washington (DC): National Academies Press (US), 2003.

22. Task Force on Surgical Palliative care; Committee on Ethics. Statement of principles of palliative care. Bull Am Coll Surg 2005;90:34-5.

23. Centers for Disease and Control Prevention NCfiPaC. Leading causes of death reports, national and regional. 1999-2015.2015. Available onlnine: https://webappa. cdc.gov/sasweb/ncipc/leadcaus10_us.html. Accessed May 11, 2020.

24. American Academy of Pediatrics. Section on Hospice and Palliative Medicine and Committee on Hospital Care. Pediatric palliative care and hospice care commitments, guidelines, and recommendations. Pediatrics 2013;132:966-72.

25. Himelstein BP, Hilden JM, Boldt AM, et al. Pediatric palliative care. N Engl J Med 2004;350:1752-62.

26. Madden K, Wolfe J, Collura C. Pediatric palliative care in the intensive care unit. Crit Care Nurs Clin North Am 2015;27:341-54.

27. World Health Organization. Cancer pain relief and palliative care in children. World Health Organization; 1998.

28. Competence Assessment Tool for Clinical Research (MacCAT-CR) for measuring children's competence to consent to clinical research. JAMA Pediatr 2014;168:1147-53.

29. Nelson LR, Stupiansky NW, Ott MA. The influence of age, health literacy, and affluence on adolescents' capacity to consent to research. J Empir Res Hum Res Ethics 2016;11:115-21.

30. Mårtenson EK, Fägerskiöld AM. A review of children's decision-making competence in health care. J Clin Nurs 
2008;17:3131-41.

31. Rowland KJ, Kodner IJ, Cashen M, et al. Surgical futility: "Aggressive" surgery on the severely demented. Surgery 2009;145:351-4.

32. Curtis JR. The use of informed assent in withholding cardiopulmonary resuscitation in the ICU. Virtual Mentor 2012;14:545-50.

33. Chang D, Parrish J, Kamangar N, et al. Time-Limited
Trials Among Critically Ill Patients With Advanced Medical Illnesses to Reduce Nonbeneficial Intensive Care Unit Treatments: Protocol for a Multicenter Quality Improvement Study. JMIR Res Protoc 2019;8:e16301.

34. Bruce CR, Liang C, Blumenthal-Barby JS, et al.

Barriers and Facilitators to Initiating and Completing Time-Limited Trials in Critical Care. Crit Care Med 2015;43:2535-43.
Cite this article as: Ott KC, Vente TM, Lautz TB, Waldman ED. Pediatric palliative care and surgery. Ann Palliat Med 2022;11(2):918-926. doi: 10.21037/apm-20-2370 